\title{
Governance, Risk und Compliance als Führungsaufgabe im Lichte der sich verändernden regulatorischen Anforderungen in der Finanzbranche am Beispiel der Schweiz
}

\begin{abstract}
Der vorliegende Beitrag untersucht, wie Führungskräfte der Finanzbranche in der Schweiz, insbesondere in der Bankenbranche, seit der Finanzkrise 2007/2008 und dem damit einhergehenden Wandel in den regulatorischen Vorschriften und der Werte allgemein mit den Themen Governance, Risk und Compliance umgehen.
\end{abstract}

\section{A. Einleitung}

Seit der globalen Finanzkrise 2007/2008 hat sich die Finanzbranche fundamental verändert. Um hohe Verluste und Insolvenzen bei Bankinstituten zu verhindern und um die gesamte Branche zu stabilisieren, wurden und werden weltweit zahlreiche neue Regulierungen eingeführt. Die Branche war vor der Finanzkrise hauptsächlich selbstreguliert. In der Schweiz gab es auf Stufe der Bundesgesetzgebung bspw. das Bundesgesetz über die Börsen und den Effektenhandel (BEH$\mathrm{G})^{1}$, das Bundesgesetz über die Bekämpfung der Geldwäscherei und der Terrorismusfinanzierung $(\mathrm{GwG})^{2}$, das Bundesgesetz über die Banken und Sparkassen $(B a n k G)^{3}$ sowie die entsprechenden Verordnungen dazu. Weiter war es die Eidgenössische Bankenkommission (EBK), die die Aufsicht über die Banken inne hatte. Seit einigen Jahren erleben wir nun global eine starke Regulierung der gesamten Finanzbranche. Neue Gesetze und Verordnungen werden im Jahresrhythmus eingeführt, in der Schweiz bspw. das Bundesgesetz über die kollektiven Kapitalanlagen $(\mathrm{KAG})^{4}$, das Bundesgesetz über die Eidgenössische Finanzmarktaufsicht (FINMAG) ${ }^{5}$ sowie das Bundesgesetz über die Finanzmarktinfrastrukturen und das Marktverhalten im Effekten- und Derivatehandel (FinfraG). ${ }^{6}$ Weiter wurde die EBK durch die Eidgenössische Finanzmarktaufsicht (FINMA) abgelöst, die nunmehr die Aufgabe zur Überwachung des gesamten Finanzmarktes ausübt. Eine Begleiterscheinung dieser Entwicklung ist, dass die Themen Governance, Risk und Compliance (GRC) damit ebenso an Wichtigkeit gewinnen: Die GRC-Einheiten werden damit beauftragt, die neusten regulatorischen Anforderungen in den Banken umzusetzen, zu überwachen und ihre Einhaltung zu kontrollieren. Nebst den Verschärfungen in der Finanzbran-

1 Bundesgesetz über die Börsen und den Effektenhandel (Börsengesetz, BEHG) vom 24.3.1995, SR 954.1.

2 Bundesgesetz über die Bekämpfung der Geldwäscherei und der Terrorismusfinanzierung (Geldwäschereigesetz, GwG) vom 10.10.1997, SR 955.0.

3 Bundesgesetz über die Banken und Sparkassen (Bankengesetz, BankG) vom 8.11.1934, SR 952.0.

4 Bundesgesetz über die kollektiven Kapitalanlagen (Kollektivanlagengesetz, KAG) vom 23.6.2006, SR 951.31

5 Bundesgesetz über die Eidgenössische Finanzmarktaufsicht(Finanzmarktaufsichtsgesetz, FINMAG) vom 22.6.2007, SR 956.1.

6 Bundesgesetz über die Finanzmarktinfrastrukturen und das Marktverhalten im Effekten- und Derivatehandel (Finanzmarktinfrastrukturgesetz, FinfraG) vom 19.6.2015, SR 958.1. 
wie Montesquieu im Mittelalter haben sich mit Machtfragen auseinander gesetzt. Insbesondere Montesquieu stellte den Leitsatz der notwendigen Gewaltenteilung auf und bezog dies nicht nur auf die Staatsorganisation, sondern formulierte allgemein als Grundgedanken, was man heute als sog. „Checks and Balances" umschreibt. ${ }^{10}$ CG fand durch die Einführung wesentlicher Bestimmungen im Aktienrecht im Jahr 1991 in der Schweiz erstmals Eingang ins OR. ${ }^{11}$ Der Swiss Code of Best Practice for Corporate Governance, der sog. „Swiss Co$\mathrm{de}^{\text {" } 12}$, zusammen mit den Richtlinien bzgl. Corporate Governance der SIX ${ }^{13}$ (RLCG) ${ }^{14}$, bilden die rechtlichen und faktischen Parameter der CG börsennotierter Unternehmen in der Schweiz und wurden im Jahr 2002 vom Schweizerische Dachverband für Wirtschaft economiesuisse genehmigt und eingeführt. ${ }^{15}$

In der Finanzbranche werden die CG-Anforderungen seit der jüngsten Finanzkrise schrittweise verschärft. ${ }^{16}$ Die wohl bekannteste rechtliche CG-Verschärfung bei Banken ist die sog. Gewaltenteilung (Verbot der Personalunion von Verwaltungsratspräsident und CEO) gem. Art. 8 Abs. 2 BankV. ${ }^{17}$ Die Aufsicht über die Banken kann als weitere Verschärfung angesehen werden. ${ }^{18}$ Die FINMA legt bei Bewilligungen sowie bei laufenden Überwachungen großen Wert auf CG-Aspekte. Die Prüfgesellschaften erstellen Risikoanalysen, in welchen CG ein zentrales Thema darstellt. Auch die Vergütungen stellen ein CG-Thema dar. ${ }^{19}$ Gemäß einer der jüngsten Medienmitteilungen der FINMA vom 1.3.2016 will sie die aufsichtsrechtlichen Anforderungen an die CG der Banken, das interne Kontrollsystem (IKS) sowie das Risikomanagement im neuen Rundschreiben 16/x - „Corporate Governance - Banken“ bündeln. Sie passt das Rundschreiben damit den Erkenntnissen aus der Finanzmarktkrise sowie den überarbeiteten internationalen Standards an. ${ }^{20}$ Namentlich zu erwähnen sind hierzu die „G20/OECD Principles of Corporate Governance“21 von November 2015, die Richtlinie „Corporate Governance Principles for Banks" ${ }^{\text {"22 }}$ des BCBS ${ }^{23}$ von Juli 2015 sowie die Empfehlungen des IWF ${ }^{24}$, welche im Rahmen des sog. „Financial Sector Assessment Program 2014“ (FSAP) ${ }^{25}$ abgege-

10 Böckli, Schweizer Aktienrecht, 4. Aufl. 2009, § 14 Rz. 16 f. m.w.N.; Iseli (Fn. 8), $\$ 1$ Rz. 29 mit Fn. 86.

11 Iseli (Fn. 8), § 1 Rz. 39 mit Fn. 120; insb. Art. 715a, 716a Abs. 1 und 2 und Art. 716b OR, vgl. Hofstetter, Corporate Governance in der Schweiz, Bericht im Zusammenhang mit den Arbeiten in der Expertengruppe „Corporate Governance“, 2002, S. 6.

12 Vgl. economiesuisse, http://www.economiesuisse.ch/sites/default/files/download s/economiesuisse_swisscode_d_web.pdf (zuletzt abgerufen am 22.5.2016).

13 Def. SIX: Swiss Exchange (Schweizer Börse).

14 Vgl. SIX Swiss Exchange, https://www.six-exchange-regulation.com/dam/downlo ads/regulation/admission-manual/directives/06_16-DCG_de.pdf (zuletzt abgerufen am 22.5.2016).

15 Böckli, Harte Stellen im Soft Law, in: Der Schweizer Treuhänder 11/2002, S. 982; Hofstetter (Fn. 11), Vorbemerkung; Iseli (Fn. 8), §1 Rz. 33

16 Kunz, Kreuzfahrt durch's schweizerische Finanzmarktrecht, in: Müller/Tschannen, Kleine Schriften zum Recht, 2014, S. 141.

17 Konkretisierung von Art. 3 Abs. 2 lit. a) BankG; Iseli (Fn.8), Rz. 546 ff.; Kunz (Fn. 16), S. 141

18 Kunz (Fn. 16), S. 141f

19 Kunz (Fn. 16), S. 142 m w. N.; Iseli (Fn. 8), Rz. 546 ff

20 FINMA, Medienmitteilung: FINMA strafft und modernisiert Anforderungen zur Corporate Governance bei Banken, https://www.finma.ch/de/ /media/finma/d okumente/dokumentencenter/8news/medienmitteilungen/20160301-mm-rs-co rpgov-d.pdf?la=de (zuletzt abgerufen am 22.5.2016).

21 Vgl. Homepage der OECD, http://www.oecd.org/corporate/principles-corporate -governance.htm (zuletzt abgerufen am 22.5.2016).

22 Vgl. Homepage des BIZ, http://www.bis.org/bcbs/publ/d328.htm (zuletzt abgerufen am 22.5.2016)

23 Def. BCBS: Basel Committee on Banking Supervision (Basler Ausschuss für Bankenaufsicht).

24 Def. IWF: Internationaler Währungsfonds (International Monetary Fund, IMF).

ben wurden. ${ }^{26}$ Die FINMA erachtet es als notwendig, zur Stärkung einer effektiven CG über die aktienrechtlichen Bestimmungen hinaus zu gehen und den Anspruchsgruppen somit grundsätzliche Informationen zum Oberleitungsorgan, zur Geschäftsleitung, zum Wahlverfahren und zur Risikopolitik zugänglich zu machen. ${ }^{27}$ Die Schweizerische Bankiervereinigung sowie economiesuisse stehen diesem geplanten Rundschreiben sehr kritisch gegenüber und versuchen, die Einführung der Neufassung zu verhindern. ${ }^{28}$ Hauptgrund dafür ist, dass CG in der Schweiz von einem erfolgreichen und etablierten System der Selbstregulierung geprägt wird, welches in die gesetzlichen Vorgaben präzise eingebettet ist. Die Empfehlungen des Swiss Code sind in allen Branchen freiwillig als Benchmark mit der Grundlage „comply or explain“ anerkannt, wobei das FINMA-RS 16/x von diesem Prinzip abweichen und den Instituten durch die neuen Vorabbewilligungsverfahren u.a. einen hohen bürokratischen und finanziellen Aufwand auferlegen würde. ${ }^{29}$

Auch eine hohe bürokratische und finanzielle Belastung würde die Trennung der Audit- und Risk-Committees insbesondere für nicht systemrelevante Banken bedeuten. Diese Trennung würde außerdem über die Empfehlungen des BCBS hinausgehen. ${ }^{30}$ Die detaillierten Regelungen bzgl. der Aufgaben der einzelnen Organe gehen ferner weiter als das OR und führen somit zu Rechtsunsicherheit. ${ }^{31}$ Inwieweit die vorgeschlagenen Änderungen nun umgesetzt werden, wird sich bei der nächsten Mitteilung der FINMA im Nachgang der Anhörungsfrist zeigen.

\section{Risikomanagement und IKS in der Finanzmarktregulierung}

Das Risikomanagement hat den Zweck, Gefahren zu vermeiden oder abzumildern, die die Unternehmensexistenz bedrohen. ${ }^{32}$ Das Risikomanagement bei Banken betrifft sämtliche Maßnahmen, die zur Verbesserung der Risikosituation eingesetzt werden können. ${ }^{33}$ Es nimmt damit eine bedeutende Stellung im strategischen Bankmanagement ein, da das Risiko per se der eigentliche Ursprung des Bankgeschäftes ist, indem die Bank darauf angewiesen ist, mit Risikoopportunitäten Erträge zu erzielen. ${ }^{34}$

25 Vgl. Homepage des IWF, https://www.imf.org/external/np/exr/facts/fsap.htm (zuletzt abgerufen am 22.5.2016)

26 FINMA (Fn. 20); FINMA, Erläuterungsbericht, Rundschreiben 2016/x „Corporate Governance - Banken“, Erläuterungsbericht (Totalrevision FINMA-RS 08/24 „Überwachung und interne Kontrollen Banken“; Teilrevision FINMA-RS 08/21 „Operationelle Risiken Banken“; Teilrevision FINMA-RS 10/1 „Vergütungssysteme“), https://www.finma.ch/de/ /media/finma/dokumente/dokumentencenter /anhoerungen/laufende-anhoerungen/rs-corpgovbanken/eb_rs_corpgovbanken 20160301 de.pdf?la=de (zuletzt abgerufen am 22.5.2016)

27 FINMA (Fn. 26), S. 17

28 SwissBanking, Prise de position sur les projets Circulaire FINMA 2016/xx „Gouvernance d'entreprise - banques“, Circulaire FINMA 2008/21 „Risques opérationnels - banques“, Circulaire FINMA 2010/01 „Systèmes de rémunération“, http://www.swissbanking.org/20160419-3020-all-stellungnahme_corporate_go vernance_version_definitiv_clean-mst.pdf, S. 1 (zuletzt abgerufen am 22.5.2016).

29 SwissBanking, Positionsbericht, S. 1f.; economiesuisse, Stellungnahme zu den FINMA-Rundschreiben 2016/x „Corporate Governance - Banken, FINMA-Rundschreiben 2008/21 „Operationelle Risiken Banken“, FINMA-Rundschreiben 2010/01 „Vergütungssysteme“, http://www.economiesuisse.ch/sites/default/file s/downloads/VNL_Finma-Rundschreiben_Corporate_Governance_DE.pdf (zuletzt abgerufen am 22.5.2016).

30 SwissBanking (Fn. 29), S. 1f

31 SwissBanking (Fn. 29), S. $1 f$

32 Wirth/Wohlmann, Zum Umgang der Unternehmer mit dem Wertewandel, in: recht - Zeitschrift für juristische Weiterbildung und Praxis 2015, S. 14 m.w.N.

33 Bröder, Risiko-Management im internationalen Bankgeschäft: Eine holistische Analyse unter besonderer Berücksichtigung der Steuerung und Kontrolle, Diss. 2006, S. $40 \mathrm{f}$ 
Eine solides internes Kontrollsystem in der Finanzbranche machen folgende Eigenschaften aus: Verantwortung der Geschäftsleitung; Kontrollumfeld; Risikoerkennung und -einschätzung; Kontrollmaßnahmen; Aufgabentrennung; Information und Kommunikation; Überwachung; Mängelbehebung sowie die Beurteilung des internen Kontrollsystems durch die Aufsichtsbehörde. ${ }^{35}$ Die Pflicht zur angemessenen internen Organisation findet sich in Art. 3 Abs. 2 lit. a) BankG sowie Art. 10 Abs. 2 lit. a) BEHG. Die Banken- und Börsenverordnungen schreiben des Weiteren eine Funktionentrennung zwischen einzelnen Geschäftsbereichen und die Erstellung eines IKS vor. ${ }^{36}$ Banken und Effektenhändler müssen über ein internes Überwachungssystem verfügen, d.h. die Aufsicht beginnt beim Institut selbst. Erreicht werden sollen damit die Wirksamkeit und Wirtschaftlichkeit von Arbeitsvorgängen und Geschäftsprozessen, die Verlässlichkeit, die Vollständigkeit und Rechtzeitigkeit der finanziellen Berichterstattung und Managementinformation sowie die Einhaltung der relevanten Gesetze, Verordnungen und Regeln. ${ }^{37}$ Im FINMA-RS 08/24 wurde die Compliance-Funktion erstmals eingehend erläutert. Heute ist sie in der Finanzbranche nicht mehr wegzudenken, um eine ordnungsgemäße und regelgetreue Geschäftsführung sicherzustellen. ${ }^{38} \mathrm{Im}$ internationalen Bereich empfiehlt das Schreiben „Compliance Function in Banks“ des Basel-Komitees $^{39}$, dass Banken ein IKS mit einer wirksamen Compliance-Funktion haben sollten. Außerdem soll das Compliance-Risiko Bestandteil des Risk Assessments sein. ${ }^{40}$

Die Arten von Risiken in der Finanzbranche bilden beim Risikomanagement und IKS einen wichtigen Faktor. Die Risikokategorien der Finanzbranche lassen sich zunächst in Primärrisiken und operationelle Risiken einteilen, woraus Reputationsrisiken folgen können. Zu den Primärrisiken gehören die Kredit-, Markt- und Liquiditätsrisiken. In den Bereich der operationellen Risiken können die Abwicklungs-, Rechts-, Sicherheits-, Haftungs-, Steuer-, Compliance- und die regulatorische Risiken subsumiert werden. ${ }^{41}$ Die eher neuen regulatorischen Risiken bezeichnen Tätigkeiten und Entscheide von Aufsichtsbehörden. Sie bilden Compliance-Risiken einer besonderen Art, weil die überwachten Akteure in einem besonderen Unterstellungs- und Kontrollverhältnis zur Aufsicht stehen. ${ }^{42}$ Ein Reputationsrisiko kann die Folge eines rechtlichen oder regulatorischen Risikos durch eine Handlung oder eine Unterlassung sein, die nicht im Gesetz stehen muss. ${ }^{43}$ Das Reputationsrisiko kann als abgeleitetes Risiko verstanden werden, denn es entsteht, wenn sich andere Risiken verwirklichen. ${ }^{44}$ In der Finanzbranche spielt dieses Risiko eine Sonderrolle, weil hier vom Einzelfall sogleich auf das System geschlossen wird - was bspw. bei Konkursen von Unternehmen

35 Roth, Good Corporate Governance: Compliance als Bestandteil des internen Kontrollsystems, Ein Handbuch für die Praxis, 2. Aufl. 2015, S. 46 Rz. 43; FINMA, Rundschreiben 2008/24, Überwachung und interne Kontrollen Banken, Überwachung und interne Kontrolle bei Banken vom 1.1.2009, https://www.finma.ch/de / /media/finma/dokumente/dokumentencenter/myfinma/rundschreiben/finm a-rs-2008-24.pdf?la=de (zuletzt abgerufen am 22.5.2016).

36 Art. 9 Abs. 1 und 4 BankV, Art. 19 Abs. 1 sowie Art. 20 Abs. 1 BEHV; Roth (Fn. 42), S. 47 Rz. 45.

37 Roth (Fn. 35), S. 47 Rz. 46.

38 Roth (Fn. 35), S. 48 Rz. 47f

39 Vgl. BIS, http://www.bis.org/publ/bcbs113.pdf (zuletzt abgerufen an 22.5.2016).

40 Roth (Fn. 35), S. 118 Rz. $201 \mathrm{f}$.

41 Bröder (Fn. 33), S. 39.

42 Roth, Compliance in a nutshell, 2. Aufl. 2015, S. 57

43 Roth (Fn. 42), S. 37; Roth (Fn. 35), S. 129 Rz. 239.

44 Bröder (Fn. 33), S. 39; Roth (Fn. 35), S. 131 Rz. 242 anderer Branchen nicht der Fall ist. ${ }^{45}$ Als Compliance-Risiko gilt das Risiko von Verstößen gegen Vorschriften, Standards und interne Vorschriften von daraus resultierenden rechtlichen und regulatorischen Sanktionen, finanziellen Verlusten und Reputationsschäden. ${ }^{46}$ Eine neuere Erscheinung stellen die sog. IT- und Cyberrisiken dar, die bei den operationellen Risiken anzusiedeln sind. Mindestanforderungen an das Konzept über die IT-Risiken umfassen u.a. eine möglichst umfassende und aktuelle Übersicht über die IT-Netzwerkumgebung bis hin zur Festlegung des Risikoappetits und der Szenarioanalysen bei Cyberattacken. Zentral ist dabei der Schutz von besonders schützenswerten Daten wie vor allem die Kundenidentifikationsdaten. $^{47}$

\section{Compliance in der Finanzbranche als umfassendes Konzept}

„Compliance“ bedeutet „Befolgung“", „Einhaltung“ oder „Erfüllung““. Der lateinische Ursprung „complere“ bedeutet „anfüllen“ oder „vollenden“. Als Teilgebiet des Risikomanagements ist der Begriff „Compliance“ als solcher eine junge Erscheinung. Der Grundgedanke, dass sich ein Unternehmen an Vorgaben zu halten und sich entsprechend zu organisieren hat, ist aber nicht neu. ${ }^{48}$ Als umfassendes Konzept beinhaltet Compliance auch allgemeine Prinzipien der Ethik und Moral, welche durch konkrete Verhaltensweisen der Mitarbeiter einfließen. ${ }^{49}$ Die Gewähr der einwandfreien Geschäftstätigkeit ist seit 1971 für die Banken in der Schweiz in Art. 3 Abs. 2 lit. c) BankG verankert. ${ }^{50}$ Die persönliche Integrität bildet dabei einen essenziellen Bestandteil. ${ }^{51}$ Der juristische Gewährsbegriff bedeutet im Kontext von Vertrauen und Reputation in funktionaler Hinsicht, dass von einer Bank erwartet wird, dass sie sich bewährt und somit dem Zweck dient, für den sie aufgebaut wurde. In sozialer Hinsicht bedeutet es, dass sich die Bank und ihre Mitarbeiter normativ und moralisch richtig und integer verhalten. ${ }^{52}$ Dies bedeutet, dass sich Banken und ihre Mitarbeiter nicht an rechts- oder sittenwidrigen Geschäften beteiligen. Die Exponenten des Bankensektors können dafür im Vergleich zu einem einzelnen Bürger sanktioniert werden. ${ }^{53}$ Die FINMA regelt die Gewährspolitik in ihrem Enforcement-Bericht. ${ }^{54}$ Der Gewährsansatz findet auch in der grenzüberschreitenden Dienstleistungserbringung seine Entfaltung, bspw. im Positionspapier der FINMA vom Oktober 2010.55 Es handelt sich dabei u.a. um Themen wie die Annahme unversteuerter Gelder aus dem Ausland und die Einhal-

45 Roth/Erni, Regulation und Reputation, Der Finanzplatz Schweiz und die Compliance seiner Unternehmen, 2008, S. $30 \mathrm{ff}$

46 Roth (Fn. 42), S. 37, 54; Bröder (Fn. 33), S. 39

47 FINMA (Fn. 26), S. 19

48 Kalberer, Compliance und Operationelles Risikomanagement, in: Roth, Close up on Compliance, Recht, Moral und Risiken - Nahaufnahme zu Compliance Management und Governance-Fragen, 2009, S. 138; Roth (Fn. 42), S. 1, 29; Wirth/Wohlmann (Fn. 32), S. 15.

49 Kalberer (Fn, 48), S. 138

50 Roth (Fn. 35), S. 153 Rz. 277; vgl. dazu Botschaft BankG 1970 (BBl. 1970 I S. 1151); Botschaft zum BankG 1934 (BBl. 1934 I S. 179 f.) sowie BankG vom 8.11.1934 (Eidg. Gesetzessammlung Band 51 (1935), S. 117 ff.).

51 Roth (Fn. 35), S. 153f. Rz. 278f.

52 Roth (Fn. 35), S. 154f. Rz. 283.

53 Roth (Fn. 35), S. 158 Rz. 294.

54 Roth (Fn. 35), S. 159 Rz. 299; FINMA, Enforcementbericht 2014 vom 24.2.2015, S. 5, https://www.finma.ch/de/ /media/finma/dokumente/dokum entencenter/myfinma/finma-publikationen/enforcementbericht/20150224-enfo rcementbericht-2014.pdf?la=de (zuletzt abgerufen am 22.5.2016).

55 Vgl. Positionspapier der FINMA zu den Rechts- und Reputationsrisiken im grenzüberschreitenden Finanzdienstleistungsgeschäft, https://www.finma.ch/de/ /me $\mathrm{dia} /$ finma/dokumente/dokumentencenter/myfinma/finma-publikationen/diskus sionspapiere/diskussionspapier-20101022-rechts-reputationsrisiken-grenzueber schr-geschaeft.pdf?la=de (zuletzt abgerufen am 22.5.2016). 
tung ausländischer aufsichtsrechtlicher Vorschriften. ${ }^{56}$ Auf internationaler Ebene behandelt das BCBS in seinen Grundsatzpapieren nicht nur Compliance-, sondern auch CG-Themen. Der Ausschuss will somit die aufsichtsrechtlichen Rahmenbedingungen der Banken stärken, eine solide Praxis im Risikomanagement unterstützen und Leitplanken für die Organisation geben. ${ }^{57}$ "Compliance and the compliance function in banks“ von April 2005 ist ein Dokument des Basler Ausschusses, das von personellen und persönlichen Anforderungen an Compliance-Mitarbeiter sowie von ihren Aufgaben handelt. ${ }^{58}$ Das Grundsatzpapier „Principles for enhancing Corporate Governance "von Oktober 2010 behandelt Fragen der Überwachung, der internen Revision, Kontrollen, RiskGovernance, Kompensationssysteme, Guidelines für den Verwaltungsrat (nachfolgend: VR) sowie Guidances für die Bankenaufsicht. Die Leitlinien hat hauptsächlich das Financial Stability Board (FSB) festgelegt. ${ }^{59}$

\section{Zusammenfassende Erkenntnisse aus Governance, Risk und Compliance für die Finanzbranche in der Schweiz}

CG ist kein neuartiger Begriff. Die Bedeutung war schon in frühen wirtschaftlichen Entwicklungen wichtig. CG findet aufgrund ihrer Wichtigkeit immer mehr Einfluss in Gesetz, Literatur und im Soft Law-Bereich. Aktuell ist eine Revision des OR im Bereich des Aktienrechts im Gange, bei welcher die CG insbesondere durch mehr Transparenz verstärkt werden soll. Es findet somit eine regelrechte Verbriefung auf höchster rechtlicher Stufe statt. Diese bringt zwar (noch) keine generelle Verpflichtung zur Erstellung eines Verhaltenskodex mit sich, erlegt aber gewissen Unternehmen eine Pflicht zur Darlegung von wichtigen Informationen über die CG im Geschäftsbericht auf - wie es heute bereits die RLCG der Schweizer Börse für börsennotierte Unternehmen vorschreibt. Für die Finanzbranche laufen zur CG große Bestrebungen, obwohl die Branche bereits heute weitreichenden Vorschriften unterliegt. Mit dem neuen FINMA-RS 16/x „Corporate Governance - Banken“ wird ein weiterer Meilenstein gelegt und die Differenzierung zwischen CG, Risk und Compliance noch mehr herausgeschält und eingeschränkt.

Der Aspekt des Risikomanagements ist unter die CG zu ordnen und macht einen wesentlichen Bestandteil einer Good Governance aus. Das Risikomanagement ist ein wichtiger Bestandteil der Finanzbranche. Aus diesem Grund müssen die unterschiedlichen Primär- und Sekundärrisiken mittels geeigneter Frameworks evaluiert werden. Die Herausforderung des Risikomanagements besteht darin, dass sich die Führung nie ganz sicher darüber sein kann, dass sie alle Risiken erkannt und auch entsprechend richtig eingestuft hat. Außerdem stellt der Mensch einen der größten und nicht beherrschbaren Risikofaktoren dar, der nie vollständig ausbalanciert werden kann. Trotz aller Regelwerke und Bemühungen muss darauf geachtet werden, dass das IKS nicht in extremis betrieben wird und eine Art Gegenreaktion damit ausgelöst wird. Die zurzeit laufenden Revisionen einiger FINMA-Rundschreiben sowie das neue Rundschreiben zur Corporate Governance werden das

. Roth (Fn. 35), S. $160 \mathrm{Rz} .301 \mathrm{ff}$

57 Roth (Fn. 35), S. 49 Rz 49

58 Roth (Fn. 35), S. 49 Rz. 50

$59 \operatorname{Roth}($ Fn. 35), S. 49f. Rz. 51.
Risiko(-management) klarer definieren und entsprechende Vorgehensweisen für die Institute festlegen.

Compliance ist eher ein neuer Begriff. Die Aufgabe der Compliance-Abteilung ist die Schadensabwendung. Compliance ist unter den Begriff des Risikomanagements zu subsumieren und führt u.a. die Kontroll- und Überwachungstätigkeit als Instrument des Risikomanagements aus. Diese Tätigkeit übt sie aber auch Hand in Hand mit den Risikoeinheiten und dem internen Audit aus, was bedeutet, dass darauf geachtet werden muss, Doppelspurigkeiten zu vermeiden. Der Beitrag der Compliance an die CG ist wichtig, aber auch limitiert, denn der Einfluss der Compliance hängt von folgenden Faktoren ab: Strategie des Unternehmens, Haltung des Top-Managements, CoC und Intensität und Qualität der Umsetzung von Vorgaben. ${ }^{60}$ Compliance kann das Verantwortungsgefühl des Managements und seinen Willen zu Integrität nicht ersetzen, denn die Compliance wird durch Mängel des Führungspersonals ausgehebelt. ${ }^{61}$ Der „tone from the top “ ist essenziell. ${ }^{62}$

\section{Faktoren, die Governance, Risk und Compliance in der Finanzbranche beeinflussen}

Die Entwicklung der GRC wird durch diverse Treiber beeinflusst, wobei GRC ihrerseits selber Einfluss auf diese Treiber ausübt. In diesem Kontext, mit Fokus auf die Finanzbranche in der Schweiz, sind drei Einflussfaktoren zu nennen: Führung, regulatorische Entwicklungen und Wertewandel. In einem ersten Schritt stehen die Themen in engem Zusammenhang mit der Führungstätigkeit, insbesondere des Top-Managements. Führung beinhaltet jedoch nicht nur die Aspekte der GRC, sondern ist auch ein ökonomisches, sozialwissenschaftliches und somit interdisziplinäres Thema. Außerdem gibt es gerade in größeren Unternehmen nicht nur das TopManagement, sondern auch die mittleren und unteren Führungsebenen, die nicht vernachlässigt werden dürfen. In einem zweiten Schritt sind GRC und die Führung den regulatorischen Entwicklungen seit der Finanzkrise 2007/2008 ausgesetzt. GRC wird immer genauer reguliert und definiert, womit die Anforderungen stetig wachsen. Dabei wird die Führung immer mehr in die Pflicht genommen. Der dritte Faktor ergibt sich aus den beiden Erstgenannten und beinhaltet den damit einhergehenden Wertewandel. Die Werte der einzelnen Themen verändern sich durch die rechtlichen und ökonomischen Entwicklungen

\section{Führung}

Führung dringt in verschiedenste wissenschaftliche Disziplinen ein. Aus diesem Grund gibt es sehr viele Modelle, Aspekte und Sichtweisen, die zu diesem Thema beachtet werden können. Die entsprechende Eingrenzung und Verortung im Kontext ist deshalb sehr wichtig. Da es sehr viele Managementsysteme gibt, ist die Führungslehre breit und beinahe unerschöpflich. Die meisten Modelle liefern Antworten auf sehr spezifische Situationen. Es bedarf aber nebst dem Werteverständnis auch des Überblicks über das Gesamte, um überhaupt ein Grundverständnis aufbauen zu können. Wichtig in

60 Roth, Kompetenz und Verantwortung: Non-Compliance als strategisches Risiko, Das Berufsbild der Compliance-Funktion und der Beitrag von Compliance an die Corporate Governance, 2012, S. 135.

61 Roth (Fn. 60), S. 135

$62 \operatorname{Roth}($ Fn. 60), S. 136 
diesem Kontext hervorzuheben ist, dass die Führung die gesamte CG eines Unternehmens ausmacht, steuert und mitgestaltet. Der wichtige kulturelle Aspekt mit all seinen Facetten gemäß Schein muss dabei mit den rechtlichen und regulatorischen Anforderungen verbunden werden. Gemäß dem neuen St. Galler-Management-Modell nach Rüegg/Grand handelt es sich um eine reflexive Gestaltungspraxis. Die Unterscheidung der Oberleitung mit der Geschäftsleitung (nachfolgend: GL) eines Unternehmens und die damit einhergehenden spezifischen Verpflichtungen werden für die Finanzbranche im neuen FINMA-RS 16/x „Corporate Governance - Banken“ explizit hervorgehoben. Dies bedeutet, dass im Rahmen der Regulierungstendenzen im Bereich GRC in der Finanzbranche auch der Bereich der Führung stark reguliert werden soll.

Wie ein Staat hat auch ein Unternehmen eine Verfassung, die jedoch nicht in sich geschlossen ist. Sie legt im Grundsatz jedoch für die selbstgewählte Rechtsform die rechtlichen Rahmennormen fest. Diese maßgeblichen Normen finden sich je nach Rechtsform im OR oder konkret im Aktienrecht, im Bankenrecht, im Arbeitsrecht, usw. Die Normen aus diesen Gesetzen bilden einen Ordnungsrahmen, der u.a. die Unternehmensorgane, deren Aufgaben, Kompetenzen und Verantwortung umfasst. ${ }^{63}$ Ein Bestandteil dieses unternehmenspolitischen Rahmens bildet die CG. Sie stellt dabei einerseits eine rechtlich determinierte, aber andererseits auch eine gestaltbare Restriktion dar. ${ }^{64}$ Nebst den gesellschaftsrechtlichen Grundregeln aus den Gesetzen gibt es in der Finanzbranche immer mehr Regelungen. ${ }^{65} \mathrm{Im}$ Bereich der CG ist, wie wir bereits gesehen haben, im Finanzmarktrecht die Einführung des Rundschreibens „Corporate Governance - Banken“ geplant. Dieses Schreiben beinhaltet auch Verschärfungen für das Oberleitungsorgan. Es wird jedoch nur soweit geboten aufsichtsrechtlich in den organisatorischen Spielraum eingegriffen. Eine Neuheit ist, dass das Oberleitungsorgan als oberster Risikohüter des Instituts definiert wird und dass der Aufgabenkatalog über die Kontrollperspektive hinaus auf sämtliche Kernkompetenzen des Aktienrechts (vgl. Art. 716a Abs. 1 OR) erstreckt wird. Alle Institute sollen sich demnach, unabhängig ihrer Rechtsform, an den aktienrechtlichen Vorschriften orientieren. Ein weiteres Novum ist, dass nebst der absoluten Anzahl Mitglieder (gem. Art. 11 Abs. 1 BankV gilt eine Mindestanzahl von drei) vor allem eine ausgewogene Zusammensetzung der Oberleitung bestehen soll. Dies bedeutet, dass das Organ genügend breit aufgestellt wird, so dass nicht nur das Wissen der Hauptgeschäftsaktivitäten vertreten ist, sondern auch die Bereiche Finanz- und Rechnungswesen, Risikomanagement, Compliance, Controlling und IT. Jedes Mitglied soll dabei über mindestens eine vertiefte Kernkompetenz verfügen. ${ }^{66}$ Gemäß diesem neuen Grundsatz der Diversität soll es in Zukunft nicht mehr möglich sein, beim Fehlen eines Prüfausschusses ein oder zwei qualifizierte VR mit spezifischen Kenntnissen mit den Aufgaben eines solchen Ausschusses zu betrauen. ${ }^{67}$ Die Diversität des VR soll auch den Geschäftsradius des Unternehmens widerspiegeln. ${ }^{68}$ Das

63 Becker, Grundlagen der Unternehmensführung, Einführung in die Managementlehre, 3. Aufl. 2015, S. $128 \mathrm{f}$.

64 Becker (Fn. 63), S. 132.

65 Becker (Fn. 63), S. 132f.

66 FINMA (Fn. 26), S. 10.

67 Vgl. FINMA, Rundschreiben 2008/24, Überwachung und interne Kontrollen Banken, Überwachung und interne Kontrolle bei Banken vom 1.1.2009, https:// www.finma.ch/de/ /media/finma/dokumente/dokumentencenter/myfinma/ru

Prinzip der Gewaltenteilung (keine Personalunion von VR und GL) wird auch weiterhin gewahrt, wobei mindestens ein Drittel des Oberleitungsorgans kein besonderes Näheverhältnis zum Institut aufweisen darf. Die Drittelregel soll eine erhöhte Verbindlichkeit haben und somit dem neuen Ansatz der Proportionalität entsprechen, da vom heutigen „comply or explain“-Ansatz ${ }^{69}$ abgekehrt wird. Die Unabhängigkeit des VR bringt auch mit sich, dass eine genügend große Distanz zu den Eigentümern besteht, d.h., der maßgebliche Teil der Mitglieder darf keine qualifizierten Beteiligungen am Institut halten. ${ }^{70}$ Bzgl. der Mandatsführung werden die Grundsätze aus den FAQ „Oberleitung von Banken und Effektenhändlern“71 der FINMA übernommen: Es wird ein aktives und zeitlich angemessenes Engagement erwartet, Interessenskonflikte sind zu vermeiden, für Krisen- und Notsituationen müssen die Mitglieder ständig zur Verfügung stehen und die speziellen Erwartungen an den prominent vertretenen Verwaltungsratspräsidenten bleiben ebenfalls bestehen. ${ }^{72}$ Wie bereits erläutert, sind die Errichtung des Prüf- und Risikoausschusses (je separat) für die größeren Banken nunmehr obligatorisch. Diese Regelung lehnt sich direkt an die Empfehlungen des BCBS an und trägt der Kritik der FSAP Rechnung, die insbesondere bei mittelgroßen Banken Defizite beim risikospezifischen Fachwissen auf Stufe der Oberleitung feststellte. ${ }^{73}$ Die Anforderungen an die Mitglieder der Prüf- und Risikoausschüsse sind die folgenden: Unabhängigkeit, fundiertes Fachwissen und Erfahrung im Spezialgebiet. Der Präsident eines Ausschusses sollte außerdem weder Präsident des Oberleitungsorgans noch eines anderen Ausschusses sein. ${ }^{74}$ Das neue Rundschreiben wird dahingehend erweitert, als dass es die wichtigsten der GL zugeteilten Aufgaben und Verantwortlichkeiten enthält: Führung des Tagesgeschäfts, Vertretung des Instituts gegenüber Dritten im operativen Bereich, Antragstellung zu Geschäften im Zuständigkeitsbereich des Oberleitungsorgans, Ausgestaltung der Führungs- und Organisationsstruktur inklusive Unterhalt eines Managementinformations- und internen Kontrollsystems sowie die operative Ertrags- und Risikosteuerung. Diese Regelung ist an sich nichts neues, da die FINMA dies bereits im Rahmen der Genehmigung der Statuten und internen Reglements verlangte. Es stellt nun jedoch eine Offizialisierung innerhalb dieses Rundschreibens dar. ${ }^{75}$ Neu beinhaltet das Rundschreiben Bestimmungen zu den Anforderungen an die Mitglieder der GL: Sie müssen gem. Art. 3 Abs. 2 lit. c) BankG einen guten Ruf genießen und Gewähr für eine einwandfreie Geschäftsführung bieten. Die GL-Mitglieder müssen somit über ausreichende Erfahrung im Bank- und Finanzbereich, Integrität, Führungskompetenz und die nötigen Fachkenntnisse ihres Funktionsbereichs verfügen. ${ }^{76}$ Der „tone from the top“ ist dabei zentral und die GL-Mitglieder müssen somit ihre Vorbildfunktion

ndschreiben/finma-rs-2008-24.pdf?la=de， Rz. $30 \quad$ (zuletzt abgerufen am 22.5.2016); FINMA (Fn. 26), S. 10.

68 FINMA (Fn. 26), S. 11

69 Def.: Das Nichteinhalten von selbstregulierenden Vorgaben muss im Geschäftsbericht erläutert werden. Mit dem Proportionalitätsprinzip wird künftig mit vorgängigen Ausnahmebewilligungen gearbeitet, vgl. FINMA (Fn. 26), S. 10.

70 FINMA (Fn. 26), S. 11

Vgl. FAQ, https://www finma.ch/de/ /media/finma/dokumente/dokumentenc enter/myfinma/faq/faq-oberleitu ng-banken-effektenhaendler.pdf?la=de (zuletzt abgerufen am 22.5.2016)

72 FINMA (Fn. 26), S. 11

73 FINMA (Fn. 26), S. 11f

74 FINMA (Fn. 26), S. 12

75 FINMA (Fn. 26), S. 12 
wahrnehmen und mit ihrem persönlichen Verhalten die Unternehmens- und Risikokultur mittragen. ${ }^{77}$

\section{Rechtliche und regulatorische Entwicklungen seit der Finanzkrise}

Die Geschehnisse seit der Finanzkrise haben dazu geführt, dass der weltweite Finanzmarkt in eine fundamentale Krise gekommen ist. Die Globalisierung, falsches Vertrauen, Unachtsamkeit, fehlende Grundprinzipien und Gesetze sowie falsches oder fehlendes Risikobewusstsein haben u.a. zu diesen Entwicklungen geführt. Durch die Aufarbeitung der Geschehnisse und die große Systemgefahr, insbesondere durch die TBTF-Institute, wurde eine regelrechte Gegenbewegung lanciert: Neue Gesetze, Regulierungen, Rundschreiben, Gremien, Gipfeltreffen, Organisationen usw. erscheinen derart engmaschig, dass es kaum möglich ist, den Gesamtüberblick zu bewahren. Da die Institute sich rechtskonform verhalten wollen und um ihre Reputation und ihre Daseinsberechtigung kämpfen, sind sie darum bestrebt, neue Vorschriften jeweils so schnell wie möglich umzusetzen. Die nationalen Entwicklungen laufen derzeit dahin, dass eine grundlegende Aktienrechtsrevision nun seit einigen Jahren pendent ist, da immer wieder neue Initiativen oder Entwicklungen hineinspielen. Auf branchenspezifischer Ebene befindet sich bspw. das FINMA-RS 16/x „Corporate Governance - Banken“ in Vorbereitung. Auf internationaler Ebene ist das Basel-Komitee federführend, unter welchem die neuen Vorschriften unter Basel III bis Ende 2018 vollständig eingeführt sein sollen. Gleichzeitig dauern die Aufräumarbeiten aus der Finanzkrise immer noch an, so dass sich die Institute einerseits um die Vergangenheitsbewältigung, andererseits um die Implementierung neuer Vorschriften zu kümmern haben.

\section{Wertewandel}

\section{Corporate Governance}

Das verstärkte Interesse an CG ist im Wesentlichen darauf zurückzuführen, dass die strafrechtliche Haftung des Unternehmens, Korruption und Privatbestechung nicht mehr nur illegitim, sondern illegal sind, und deshalb staatlich sanktioniert werden. Diese Änderung der Wahrnehmung zeigt, dass in der Unternehmensführung nicht mehr das Credo „alles ist möglich“ gilt und es Kavaliersdelikte nicht mehr gibt. ${ }^{78}$ Wie bereits ausgeführt, ist der „tone from the top“ essenzieller Bestandteil einer guten CG. Nur, wenn die oberste Führungsstufe die eigenen Werte vorlebt und weitergibt, haben die CGThemen eine Chance, im Unternehmen verinnerlicht zu werden. Es ist daher zu empfehlen, die Chancen und Risiken aus dem Wertewandel in einem Code of Conduct festzulegen, wobei auch die Kommunikationskanäle deutlich aufzuzeigen sind. Dies ist jedoch nicht immer der Fall. Vielfach befinden sich auf der obersten Stufe Führungskräfte, die sich auf ihre jahrzehntelange Erfahrung berufen, anstatt ihren Mitarbeitern „zuzuhören“. Sie weigern sich bspw., Experten beizuziehen und wehren sich gegen neue Entwicklungen. Gerade um Veränderungen rechtzeitig begegnen zu können, ist dieses „Zuhören“ wichtig. Wenn das Top-Management dazu nicht bereit ist, wirkt sich das auf die nachfolgenden Stufen aus. Die mangelnde Bereitschaft, sich mit neuen Fragestellungen, sei es in
Bezug auf Produkte, Märkte, Technologien oder auch Werte auseinanderzusetzen, stellt für viele Unternehmen die größte Gefahr dar. ${ }^{79}$ Globale Treiber der CG-Bewegung sind die Globalisierung der Wirtschaft und die Liberalisierung der Kapitalmärkte, da die international tätigen Akteure des Kapitalmarktes den Governance-Modalitäten mit Nachdruck Beachtung schenken. Antrieb erhält die Diskussion um effiziente und transparente Formen der Unternehmensführung auch durch Fälle von Missmanagement und Unternehmensschieflagen. Aus diesen Gründen werden im Rahmen der Aufarbeitung der jüngsten Finanzkrise bisherige Governance-Modalitäten in den letzten Jahren konsequent in Frage gestellt. ${ }^{80}$

\section{Risk und Compliance}

Ein Wertewandel führt regelmäßig zu Chancen und Gefahren für die Unternehmenstätigkeit. Eine frühzeitige Erkennung durch das Risikomanagement ist deshalb essenziell. Ab wann Wertewandel jedoch für das Risikomanagement ersichtlich sind, ist ungewiss. Das Risikomanagement ist ein kontinuierlicher Prozess, was bedeutet, dass die Risikoidentifikation sowie die anschließende Beurteilung kontinuierlich stattzufinden haben und regelmäßig überprüft werden müssen. ${ }^{81} \mathrm{Im}$ Rahmen des strategischen Risikomanagements ist es die Aufgabe des VR, ein Früherkennungssystem zu etablieren, um Wertewandel zu erkennen, einzustufen und frühzeitig Maßnahmen zu ergreifen. ${ }^{82}$ Eine strategische Antwort auf diesen Wertewandel oder Paradigmenwechsel liefert Kurer. Seines Erachtens sind insbesondere die rechtlichen Risiken lawinenartig angewachsen und deshalb braucht es hauptsächlich drei Aspekte: Die Risiken sollen erstens nicht mehr nur von den Juristen, sondern vom VR und der GL selber gesteuert werden. Zweitens sollen sie den Prozess zur Kontrolle der Rechts- und Compliance-Risiken als unternehmerischen Kernprozess verstehen und drittens sollen gezielte Tools eingesetzt werden, die das Führen der Risiken erleichtern und unterstützen. $^{83}$ Ein solches Tool wäre z.B. eine sog. „Roadmap to Legal Risk Management ${ }^{\text {“84 }}$, wobei das Management durch einen iterativen Prozess geht, der aus sechs Elementen besteht: Definition der Ursachen der spezifischen Risiken, Treffen organisatorischer Vorkehrungen, um die Strategie umzusetzen (z.B. durch ein Legal Risk Framework), Gestaltung der geeigneten Operationen und Prozesse (z.B. Etablierung eines Compliance-Programms), Prüfung des Einsatzes gezielter Technologien, sowie kostengünstige und wirkungsvolle Führung der Risiken (z.B. Big Data, künstliche Intelligenz, FinTech und Robotik). ${ }^{85}$ Dieser technologische Schritt geht aber auch in die Richtung, dass gewisse Arbeiten von Juristen und Compliance-Abteilungen durch künstliche Intelligenz ersetzt werden können. ${ }^{86}$ In einem letzten Schritt geht es auch um die kulturelle Dimension der Risiken. Der Steuerung des menschlichen Verhaltens soll mehr Aufmerksamkeit geschenkt werden, da die meisten rechtlichen Großrisiken auf

79 Wirth/Wohlmann (Fn. 32), S. 17.

80 Werder, Führungsorganisation, Grundlagen der Corporate Governance, Spitzenund Leitungsorganisation, 3. Aufl. 2015, S. 5f.

81 Wirth/Wohlmann (Fn. 32), S. 14 m.w.N

82 Wirth/Wohlmann (Fn. 32), S. 15 m.w.N

83 Kurer, Wenn das Paradigma wechselt: Rechtsrisiken in der globalen Welt, in: GesKR 2015, 333.

84 Kurer, Legal and Compliance Risk, A Strategic Response to a Rising Threat for Global Business, 2015, S. 9f

85 Kurer (Fn. 84), S. 333f.

86 Kurer (Fn. 84), S. 334; Kurer, Der Jurist im digitalen Holozän, NZZ Feuilleton vom 19.4.2016, S. 36. 
unethisches Verhalten und menschliches Versagen zurückzuführen sind (sog. Verhaltensrisiken). ${ }^{87}$ Das Risikomanagement muss heute strategisch betrieben und von der Unternehmensspitze aus gesteuert werden, so dass Juristen nicht mehr die einzigen sind, aber für ihr normatives Wissen dennoch konsultiert werden müssen. ${ }^{88}$ Die Verknüpfung der Veränderung in der Unternehmenskultur mit der Veränderung der Gesetzgebungskultur führt somit zu den heutigen Compliance-Organisationen. Das was heute gilt, kann morgen bereits nicht mehr zulässig sein. Der Wandel vollzieht sich rasant, worauf die Unternehmen reagieren müssen. ${ }^{89}$

Mit dieser Entwicklung wurde auch die Nachhaltigkeit von Compliance als Thema etabliert. Gute Compliance-Tätigkeit zeichnet sich nicht nur dadurch aus, die aktuellen Gesetze einzuhalten, sondern auch, die künftigen Regelungen zu beobachten. Je prominenter eine Branche oder ein Geschäft in der Politik und in den Medien vertreten ist, desto intensiver sollten die Tendenzen und Entwicklungen der Moralvorstellungen, die die Gesetzgebung der nächsten Jahre beeinflussen könnten, verfolgt werden. ${ }^{90}$ Bei der Aktienrechtsrevision von 1992 war Compliance noch ein unbekanntes Wort. Die Rechtsabteilungen von Unternehmen hatten immerhin die Nebenaufgabe, die Erfüllung und Einhaltung der Gesetze sicherzustellen, aber eigentliche Compliance-Organisationen gab es nicht ${ }^{91}$. Drei Entwicklungen sind zu nennen, die dazu führten, dass die heutigen Unternehmen über nicht mehr wegzudenkende Compliance-Organisation verfügen: Der geänderte Umgang mit „Gentleman-Delikten“, die starke Zunahme der Regulierungsdichte und die Zunahme der Unternehmenszusammenschlüsse. ${ }^{92}$ Kritisch betrachtet wird dieser Wertewandel insbesondere durch die Medien. Die Finanzbranche wird durch neue Regeln derart eingeschränkt, dass sich die Bankangestellten an die Vorschriften halten und den „gesunden Menschenverstand“" aus Angst vor Verstößen nicht mehr anwenden. ${ }^{93}$

\section{Führung}

Die oberste Führungsebene eines Unternehmens trägt die Verantwortung für die frühzeitige Erkennung eines Wertewandels und den entsprechenden Umgang damit. Diese Verantwortung liegt jedoch nicht alleine beim Top-Management. Entscheidend dafür sind auch die Organisation der Gesellschaft sowie die Unternehmenskultur. Ferner müssen etablierte und gute Kommunikationswege vorhanden sein, so dass Erkenntnisse entsprechend platziert werden können. ${ }^{94}$ Neue Regulierungen und Gesetze können helfen, aber die Forderung nur begrenzt umsetzen und allenfalls auch ein falsches Sicherheitsgefühl hervorrufen. Dies führt zu einer Lücke in der Erwartungshaltung zwischen Norm und Wirkung. Von einer Führungspersönlichkeit wird daher erwartet, dass sie nicht bloß zwingende gesetzliche Normen einhält, sondern dass sie in der Lage ist, ethisches und nachhaltiges unternehmerisches Denken als Leitplanke zu nehmen. ${ }^{95}$ Es ist daher wichtig, ver-

87 Bröder (Fn. 33), S. 206; Kurer (Fn. 84), S. 334; Roth/Erni (Fn. 45), S. 53 ff

88 Kurer (Fn. 84), S. 334f.

89 Wirth/Wohlmann (Fn. 32), S. 16 f. m.w.N

90 Wirth/Wohlmann (Fn. 32), S. 16 f. m.w.N.

91 Roth (Fn. 42), S. 1; Wirth/Wohlmann (Fn. 32), S. 15 m.w.N

92 Wirth/Wohlmann (Fn. 32), S. 15 f. m.w.N.

93 Imwinkelried, Paragraph sowieso, NZZ vom 18.3.2016, S. 12

94 Wirth/Wohlmann (Fn. 32), S. 17 mw.N

95 Wirth/Wohlmann (Fn. 32), S. 18 m.w.N.

änderte Werte nicht einfach in gesetzliche Normen umzusetzen, sondern vermehrt auf die Sorgfalts- und Treuepflicht zu verweisen. ${ }^{96}$ Durch die aktuellen CG-Bewegungen gewinnen die Gestaltungsoptionen der Führungsorganisation und ihre Effizienzwirkungen an Stellenwert. ${ }^{97}$ Dies zeigt auch die Studie zu den Führungsherausforderungen von morgen, wo beim Trend zur sozialen Verantwortung und Nachhaltigkeit die CG einen wichtigen Aspekt darstellt. ${ }^{98}$

\section{Regulatorische Entwicklungen}

Gemäß Kotz ist das Resultat der Regulierungstendenzen in den USA, dass es seit dem Dodd-Frank-Act überlappende Zuständigkeiten gibt. Es bestehen bei den Finanzinstituten Unsicherheiten darüber, welche Behörde nun für ein bestimmtes Finanzprodukt zuständig ist. ${ }^{99}$ Es bestehen aber nicht nur Unsicherheiten in Bezug auf die Produkte, sondern auch in Bezug auf den Markt und die Marktteilnehmer. ${ }^{100}$ Des Weiteren gibt es in den USA trotz der regulatorischen Entwicklungen immer noch Kollapse von Finanzinstituten. ${ }^{101}$ Noch ein weiterer Punkt, der ausgebaut werden sollte, ist die Verbesserung der Koordination zwischen den regulatorischen Behörden. Es wurde zwar der FSCO (Financial Stability Oversight Council) im Nachgang des Dodd-Frank-Acts eingeführt; dieser fokussiert jedoch mehr die breiten Themen des Finanzmarktes und geht weniger auf die täglichen Anforderungen der regulatorischen Behörden ein. ${ }^{102}$ Als zusätzlicher Punkt ist es wichtig, dass die Institute verstehen, dass sich das regulatorische Klima in der nahen Zukunft sehr wahrscheinlich nicht ändern wird. Die Unternehmen müssen deshalb die notwendigen Werkzeuge zur Verfügung haben, um mit den überlappenden Anforderungen fertig zu werden. Außerdem müssen sie den Sinn und Zweck jeder einzelnen Regulierung verstehen und auch Offenheit gegenüber dem Regulator zeigen. Nur mit einer guten Zusammenarbeit können die gemeinsamen und abweichenden Interessen verfolgt werden. ${ }^{103}$ Die fehlende Kooperation ist nicht nur in den USA spürbar, sondern auch allgemein im internationalen Bereich. Es entsteht insbesondere Druck auf die Kooperation der Regulierungen der einzelnen Staaten. ${ }^{104}$ Ob Regulierung und Aufsicht die Subprime-Krise hätten verhindern können, ist umstritten. Eine Theorie dazu ist, dass Auslöser, Geschwindigkeit, Ausmaß und Dauer der Ereignisse sehr überraschend waren und der entscheidende Punkt der Umgang des Top-Managements mit den Risiken ist. ${ }^{105}$ Die Regulierung kann sozusagen auch als Reputationsfaktor gesehen werden, indem Institute zwar den entsprechenden Standards zustimmen, diese intern aber nicht konsequent durchsetzen. ${ }^{106}$

Ein aktuelles Phänomen, das durch die Fülle an internationalen Standards entsteht, ist der Wettbewerb zwischen internationalen Standards, der sich zwischen den Staaten und auch

\footnotetext{
96 Wirth/Wohlmann (Fn. 32), S. 18 m.w.N

97 Werder (Fn. 80), S. 26

98 Vgl. dazu Eberhardı/Majkovic, Die Zukunft der Führung: Eine explorative Studie zu den Führungsherausforderungen von morgen, 2015, S. 38.

$99 \mathrm{Kotz}$, Financial Regulation and Compliance - How to Manage Competing and Overlapping Regulatory Oversight, 2015, S. 206.

$100 \mathrm{Kotz}$ (Fn. 99), S. 207

101 Kolz (Fn. 99), S. 209

102 Kotz (Fn. 99), S. 210f.

103 Kotz (Fn. 99), S. $212 \mathrm{f}$

104 Wandel, International Regulatory Cooperation: An Analysis of Standard Setting in Financial Law, Diss. 2014, S. 18, $194 \mathrm{ff}$

105 Roth/Erni (Fn. 45), S. 36
} 
zwischen den internationalen Organisationen austrägt. Informationsasymmetrien und negative externe Effekte führen gemäß der Wirtschaftswissenschaft zu Marktversagen. ${ }^{107}$ Falls deshalb der freie Wettbewerb auf den Finanzmärkten aufgegeben werden muss, ist klar, dass sich im Wettbewerb der Rechtssysteme das bessere politische System und das optimalere Regulierungsniveau durchsetzen. Die Staaten ringen um nationale Vorteile und Nischen. ${ }^{108}$ Dem regulatorischen Konkurrenz-Modell wird jedoch mittels Koordination entgegen zu halten versucht - insbesondere durch Bemühungen der G20, des FSB, der IOSCO, der IASB sowie des FSAP. ${ }^{109}$ Aufgrund von Inkonsistenzen in den EU- und US-Regeln kommt es in der weltweiten Finanzmarktregulierung zu Arbitrage, was wiederum dazu führen kann, dass die Staaten vermehrt protektionistische Politik betreiben und sich somit die grenzüberschreitenden Dienstleistungen verteuern. ${ }^{110}$ Auch, wenn der Wettbewerb zu Vorteilen führen kann, muss dennoch die Balance zwischen der Bereitstellung praktikabler und gleicher Wettbewerbsbedingungen und einer ausreichenden Flexibilität für die Besonderheiten nationaler Finanzsysteme bestehen. ${ }^{111}$ Die IOSCO hat ein sog. „Regulatory Toolkit“ errichtet, um dem Spannungsfeld zwischen Koordination und Wettbewerb in grenzüberschreitenden Sachverhalten entgegen zu wirken. ${ }^{112}$ Die Setzung der internationalen Standards bedeutet bzw. bewirkt, dass eine Fülle an Regulierungen im Bereich des Soft Law entsteht, da weltweit keine demokratisch legitimierte Abstimmung über Gesetze gemacht werden kann. Die Legitimation beruht somit auf den Vertretern der einzelnen Staaten, Welt- und Nationalbanken in den entsprechenden Gremien und wird in den einzelnen Staaten durch die Adaptierung in die staatlichen Gesetze vollzogen. Gültigkeit haben die Standards aber auch dann, wenn sie von den einzelnen Staaten nicht explizit in ihre Gesetzeslandschaft aufgenommen werden. ${ }^{113}$ Aus diesem Grund ist in den meisten Fällen kein rechtliches Mittel vorhanden, Institute bei fehlender Umsetzung internationaler Standards zu ahnden. Somit wird versucht, die Einhaltung der Standards durch sog. „Regulatory Reviews“ oder „Assessments“ (bspw. FSAP) zu bewirken, was mit einer Art „Selbstverstaatlichung der Privatwirtschaft“ ohne demokratische Legitimation verglichen werden kann. Ein weiteres wichtiges Mittel ist jedoch das intrinsische Streben nach Reputation, denn nur, wenn die Adressaten der Regulierung wie auch die Regulatoren ausreichend motiviert sind, lässt sich das Erwünschte erreichen. ${ }^{114}$ Kurer erkennt in dieser Tendenz von justiziablen Rechtsregeln zu programmatischen Aspirationen, dass sich das Recht allmählich wie „Zucker in der Teetasse“ aufzulösen droht und somit die Urteilskraft von Juristen bald nicht mehr gefragt sein könnte. ${ }^{115}$

Gemäß Kotz gibt es fünf Kriterien, die zur Stärkung von GRC im sich verändernden regulatorischen Umfeld beitragen: Offenlegung der vollständigen Vergütung der Geschäftsleitung, Implementierung effektiver Grundsätze und Prozesse, Schaffung von Verantwortung innerhalb einer Organisation, Erken-

107 Weber, SZW 2015, 579.

108 Weber, SZW 2015, 579 (579 f.)

109 Weber, SZW 2015, 579 (581).

110 Weber, SZW 2015, 579 (581)

111 Weber, SZW 2015, 579 (581)

112 Weber, SZW 2015, 579 (583)

113 Wandel (Fn. 104), S. 12

114 Weber, SZW 2015, 579 (585)

115 Kurer (Fn. 100). nen von Warnsignalen in einer unethischen Kultur (Abschieben von Verantwortung, unseriöse Äußerungen, Machtkämpfe) und Willensbildung auf ethischem Fundament. ${ }^{116}$ Wie man erkennen kann, sind die Themen derart miteinander verknüpft, dass Abgrenzungen kaum möglich sind. Eine der Erkenntnisse ist, dass es ein regelrechtes Zusammenspiel der GRC untereinander gibt. Dieses übt ihrerseits einen starken Einfluss auf die Führungsaufgabe aus und wird selber durch die Entwicklungen der Regulierungen und des Wertewandels vorangetrieben, so dass ein regelrechtes Kräfteverhältnis entsteht (vergleichbar mit den sog. „Five Forces“ von Porter).

\section{Erkenntnisse aus der Praxis}

Die theoretischen Erkenntnisse (A.-C.) wurden durch dreizehn qualitative Interviews mit Führungskräften vom Bankenplatz Zürich auf ihre Praxistauglichkeit hin geprüft. Die wichtigsten Erkenntnisse darauf werden im Folgenden dargestellt.

\section{Schlüsselaussagen aus der Praxis}

Die Übernahme des inflationären Bussenwesens aus dem angelsächsischen Raum erachten viele Führungskräfte als keine gute Entwicklung für die Schweiz. Der vorauseilende Gehorsam, respektive die Umsetzung regulatorischer Erfordernisse innerhalb der Institute, gehen für viele Interviewpartner zu weit. Dies sei vor allem dann problematisch, wenn sich die neuen Auflagen erst in der Anhörungsphase befinden oder noch nicht in Kraft getreten sind. Außerdem erhöhe dies die Komplexität, da immer geschaut werden muss, ob die Basis nun eine internationale Vorgabe, ein nationales Bundesgesetz, eine nationale RL oder ein sog. „institutsspezifischer Finish" darstellt.

Zudem sind einige Führungskräfte auch der Ansicht, dass es nicht für jede neue Problematik ein Gesetz braucht. Dies ist jedoch Auswuchs dessen, dass in der Branche nun viele nicht demokratisch legitimierte „Gesetze der FINMA“ erlassen werden. Die Wirkungsdiskussionen müssten dabei zwingend durch Branchenvertreter, und nicht durch Juristen geführt werden. Diese Entwicklung nähme dann eine zusätzliche unüberschaubare Dimension an, wenn man gemäß Aussage einer Führungsperson bedenkt, dass mit ca. 150 neuen Regulierungen pro Tag und somit ca. 40.000 neuen Vorschriften pro Jahr eine regelrechte Regulierungsflut herrscht. Viele Führungskräfte aus dem Frontbereich oder aus frontnahen Bereichen sind der Ansicht, dass ein Überformalismus herrsche, der nicht mehr gesund ist. Außerdem arteten viele vermeintliche Risikokontrollen aus, so dass am Ende des Tages bei den Kontrollen nicht mehr die eigentlichen Risiken im Vordergrund stünden, sondern gewisse Nulltoleranzen an Themen eingeführt würden, weil man ein gewisses Verhalten einfach nicht mehr dulden will (sog. Management by NOK). Die Balance zwischen Risk/Return gelte es dabei jedoch nicht aus den Augen zu verlieren.

Bemerkenswert bei der Diskussion über die Risiken ist auch, dass die eigentlichen Kern- oder Primärrisiken einer Bank, die u.a. aus dem Kreditgeschäft herrühren, relativ unproblematisch und unter Kontrolle seien. Viele Führungskräfte äußern, dass gerade die Ausfallbeträge in diesem Risikobereich relativ gesehen eher klein und überschaubar sind, wohingegen 
bei den sekundären oder Folgerisiken (Compliance- oder operationelle Risiken) mit ungewissen Kosten zu rechnen ist. Es ist festzustellen, dass insbesondere Führungskräfte aus dem frontnahen oder Frontbereich die Aktivitäten innerhalb der GRC-Themen eher als reaktiv, wohingegen GL-Mitglieder oder GL-nahe Führungskräfte das Institut als proaktiv agierend empfinden. Eine Führungskraft betont, dass in den ganzen regulatorischen Entwicklungen oftmals die sog. „Firstmovers" bestraft werden. Das sind diejenigen Institute, die z.B. zuerst neue Vorschriften umsetzen und z.B. damit einhergehend gewisse Produkte nicht mehr anbieten. Der Regulator stellt dann fest, dass das Vorgehen bspw. unwirtschaftlich oder nicht praktikabel ist, und lockert dann die Vorschriften wieder. Der Firstmover hat dann das Nachsehen, weil er dadurch schon geminderte Erträge hat oder gewisse Kundenbeziehungen nicht mehr vorhanden sind.

Bei den Anreizsystemen zur Einhaltung von GRC driften die Meinungen stark auseinander. Je nach Geschäftseinheit oder Institut herrscht eine andere Kultur. Gewisse Institute haben die Anforderungen in ihre Qualifikationen der Mitarbeiter[SC1] und Führungskräfte implementiert (dies wird dann, je nach dem, als gut oder suboptimal empfunden). In gewissen Instituten ist dieser Teil dann auch bonusrelevant. Andere Institute hingegen, in der Tendenz die kleineren, implementieren solche „Selbstverständlichkeiten“ nicht in die Qualifikationen. Vereinzelte Führungskräfte erwähnen, dass das sog. das Change-Management heute eine Grundhaltung jeder Führungsperson sein muss. Im Vergleich zu früheren Führungsgenerationen sei dies eine gewaltige Entwicklung, mit welcher viele ältere Führungskräfte nicht mehr Schritt halten können. Viele Führungskräfte, vor allem diejenigen aus dedizierten GRC-Einheiten, bemerken, dass sich die täglichen Aufgaben der Juristen, Compliance- und Risk-Officers extrem verändert haben. Sie waren früher für die Front und auch andere interne Bereiche beratend tätig. Heute aber müssen sie multitaskingfähig sein, insbesondere fundiertes Spezial- und Prozesswissen haben sowie Projekterfahrung aufweisen. Für die Juristen und Anwälte ist vor allem die Bandbreite an „EnforcementMöglichkeiten“ größer geworden, d.h. die Bank wird nicht nur aus finanzmarktrechtlichen Vorschriften zur Rechenschaft gezogen, sondern bspw. auch aus wettbewerbsrechtlichen.

Die Kommunikation, respektive die Adressierung von Themen von oberen Führungsstufen nach untere Führungsebenen, sei einfacher als diejenige von der unteren oder mittleren Ebene zum Top-Management. Gründe dafür seien, dass die Informationen von „unten“ nach „oben“ kurz aber möglichst vollständig sein sollen (Details gehen verloren) und Ängste, dass die Adressierung eines Problems als Unfähigkeit des unteren Managements betrachtet werden könnte. Gemäß vieler Führungskräfte ist die IT-Stabilität zur Verhinderung von $\mathrm{Cy}$ ber-Attacken sowie um das Tagesgeschäft sicherzustellen sehr wichtig geworden; diese stellten ebenso ein großes operationelles Risiko dar. Mit der kommunikativen Vernetztheit treten auch Themen wie „Social Engineering““ vermehrt in den Vordergrund. Eine Führungsperson betont das politische und mediale Umfeld hinter der ganzen Entwicklung. Nach der Finanzkrise seien viele Institute relativ schnell wieder finanziell stabil gewesen, aber die Reputation war die große Herausforderung und auch der Grund, weshalb viele Kundengelder abflossen und sich damit neue Herausforderungen für die Insti-

tute ergeben hätten. Auch die mediale Berichterstattung, z.B. über Fälle wie die „Panama-Papers“, könnte großes Misstrauen gegenüber allen Instituten wecken, obwohl sehr viele Institute keine sog. „Panama-Fälle“ haben. Obwohl insbesondere die großen und weltweit tätigen Finanzinstitute im Fokus des öffentlichen Interesses stünden, sind die kleineren Institute gemäß einer Führungsperson genauso stark von den GRCThemen betroffen wie die großen: GRC verlaufe nämlich mit der Institutsgröße nicht degressiv, sondern linear.

Die Kultur und den einzelnen Menschen erwähnen fast alle Führungskräfte im Zusammenhang mit GRC und den regulatorischen Entwicklungen. Das Vertrauen in die Menschen spiele dabei mehr denn je eine wichtige Rolle. Eine gewisse Fehlerkultur wird auch aktiv gelebt und manche Fehler werden nicht bestraft. Deshalb müsse die Optik der Risiken hinterfragt werden und die Fehler müssten zwingend dem korrekten Verhalten gegenübergestellt werden, d.h. auch die guten und korrekten Dinge müssen aktiv hinzugezogen werden, damit ein fairer Vergleich gemacht werden kann. Dies geht auch mit der Verankerung der Sinnhaftigkeit einher: Viele Führungskräfte erwähnen, dass viel Kommunikation und Aufklärung zu den Themen notwendig ist. Dies einerseits, um das korrekte Verhalten herbeizuführen, und andererseits, um das Verständnis und die Zusammenhänge für die Themen zu fördern. Insbesondere bei den Kundenberatern ist dies extrem wichtig, denn diese führen die Gespräche mit den Kunden und müssen ihnen gegenüber auch erklären, weshalb gewisse Dinge nun zusätzlich notwendig sind. Es sei mit der Fülle an Vorschriften und Zurechtweisungen auch wichtig, dass den Menschen nicht das eigene Denken, das Augenmaß und der gesunde Menschenverstand abhandenkommen. Der einzelne Mensch stellt deshalb andererseits auch ein großes sog. „tail risk“117 dar: Eine einzelne Person kann mit 10.000 Kundendaten aus der Bank gehen und somit das Institut extrem schädigen. Deshalb ist Fingerspitzengefühl bei jedem einzelnen Mitarbeiter gefordert.

\section{Mögliche Handlungsmaßnahmen aus der Praxis}

Aus den Gesprächen mit den Führungskräften haben sich einige Herausforderungen und Stolpersteine, insbesondere bzgl. der laufenden Regulierungen und GRC allgemein herauskristallisiert, aus welchen sich die folgenden Handlungsmaßnahmen für den Regulator, die Unternehmen und die Führungskräfte ableiten lassen:

- Regulatorische Entwicklungen auf multinationaler, nationaler und innerbetrieblicher Ebene alignieren;

- Überformalismus vor allem in der innerbetrieblichen Umsetzung der Regulierungen vermeiden;

- Fokussierung der Kontrollmaßnahmen auf die richtigen und wichtigen Risiken;

- Bewahrung der Autonomie und des Wettbewerbs in der freien Marktwirtschaft trotz aller Risiken;

- Umdenken der Führungskräfte und Mitarbeiter vermehrt durch Kulturwandel anstelle von angelsächsischen Sanktionierungstendenzen;

117 Def.: Risiko eines Extremereionisses. 
- Erkenntnisse aus anderen Wissenschaften (z.B. Neurowissenschaften) nutzen, um die systemunterstützten Kontrollmechanismen gezielt auf das menschliche Verhalten zuzuschneiden.

\section{E. Schlussfolgerung und Ergebnisse}

\section{Wie gehen die Führungskräfte der} Finanzbranche mit GRC im sich verändernden regulatorischen Umfeld um?

Governance, Risk, Compliance, das regulatorische Umfeld und der Wertewandel beeinflussen die Führungstätigkeit in der Finanzbranche fundamental. Die Führung wird vor neue inhaltliche Herausforderungen gestellt, etwa neue Gesetze oder Rundschreiben der FINMA, und befindet sich dabei gleichzeitig in einem fundamentalen Veränderungsprozess der gesamten Branche. Es gilt zu unterscheiden, welche Führungspersonen, d.h. welche Hierarchiestufen eines Unternehmens, betrachtet werden: Die Oberleitung (i.d.R. der VR) nimmt die Vogelperspektive im Unternehmen ein und trägt die Hauptverantwortung. In ihrer Funktion kann und soll sie operativ nicht alles kontrollieren, ist auf die einwandfreie Geschäftsführung durch die GL angewiesen und vertraut auch darauf.

Die GL ist mit einer wachsenden Zahl inhaltlicher Themen konfrontiert und nimmt im Zuge der laufenden regulatorischen Entwicklungen eine Vermittlerrolle zwischen den Banken und den Behörden wahr, um das System in eine für die Behörden und die Institute gangbare und praktikable Richtung zu lenken. Sie formt zusammen mit der Oberleitung die CG, worin sie u.a. die Kultur sowie die Art und Frequenz der Kommunikation festlegen, um für Transparenz gegen innen und außen mit den Anspruchsgruppen und vor allem gegenüber den Behörden sorgen. Sie ist damit auch mit der eigentlichen Einhaltung, Überwachung und Implementierung der regulatorischen Vorschriften betraut und kann aus ihrem
(Vor-)wissen hinaus proaktiv agieren und beeinflussen. Ihr Anreiz besteht dabei darin, im Innenverhältnis ein gemeinsames Werteverständnis im ganzen Institut herbeizuführen und im Aussenverhältnis gegenüber den Rahmenbedingungen und dem Regulator die notwendigen Vorschriften einzuhalten, damit die Existenz des Instituts gewährleistet und das wirtschaftliche Fortkommen sichergestellt ist.

Das mittlere und untere Management ist in der Folge mit den Entscheidungen der GL konfrontiert und agiert gerade im Bereich Risk und Compliance. Die Kommunikation und das Erklären über die Entwicklungen sind essenziell, damit die Kontrollen im Rahmen des IKS und der Compliance erfolgreich ausfallen und der interne sowie in der Folge der externe Audit keine Auffälligkeiten entdeckt. Im Rahmen ihres Wissenstandes, ihrer Aufgaben, Kompetenzen und Verantwortung agieren sie in der Umsetzung der Vorgaben der GL meist reaktiv. In der Erkennung von fehlerhaftem IKS oder beschlossenen Grundsätzen, die sich im operativen Bereich fast nicht umsetzen lassen, sollen sie proaktiv agieren und diese Informationen in die hierarchisch übergeordneten Gremien tragen. Die Kommunikation von „oben“ nach „unten“ erweist sich jedoch als einfacher, als diejenige von „unten“ nach „oben“. Ihr Anreiz ist es, die Risiken als Grundhaltung und Bestandteil des Geschäfts unter Kontrolle zu haben und keinen Schaden für die Bank herbeizuführen. Die meisten Institute benutzen als Mittel oder Evidenz dazu diverse Reportings der R\&C-Einheiten sowie die jährlichen Qualifikationsziele jedes einzelnen Mitarbeiters.

\section{Ist und bleibt GRC eine Führungsaufgabe unter dem regulatorischen Wandel der Finanzbranche?}

Corporate Governance ist im Zuge der Finanzkrise 2007/ 2008 eine immer wichtiger werdende Führungsaufgabe geworden. Der Begriff kann als neuer Ausdruck für „Führung“ oder "Management" erachtet werden und bildet den Grundstein für die Oberleitungsorgane und die GL. Das Top-Management ist bei den schnellen Änderungen des momentanen regulatorischen Umfeldes auf Informationsträger und -zulieferer angewiesen und lässt sich mehr denn je von GRC-Experten beraten und Handlungsoptionen unterbreiten. Die Verantwortung und die Entscheidungsmacht liegen nach wie vor beim Oberleitungsorgan und der GL. Das Knowhow kann durch das Top-Management alleine jedoch nicht abgedeckt werden. Dies spiegelt sich auch auf der mittleren und unteren Führungsebene wider: Sie sind in ihren Gremien und Führungssitzungen auf Experten aus dem R\&C-Bereich angewiesen, um Einzelfallentscheidungen zu fällen und die $\mathrm{Ge}$ - 
samtzusammenhänge zu sehen. Das breite und tiefe Knowhow über die GRC-Themen und das regulatorische Umfeld kann als kritischer Erfolgsfaktor der heutigen Führungspersonen in der Finanzbranche erachtet werden.

\section{These 1: Die Verantwortung über GRC wird den dedizierten GRC-Einheiten übertragen}

Dass die Verantwortung über GRC faktisch bei der Führung liegt und bleiben wird, ist in der Lehre und gemäß Experten unbestritten, und wird auch mehr denn je gefordert und mit Maßnahmen im Vergütungssystem forciert. In der Praxis kristallisiert sich jedoch heraus, dass die Entscheidungen der Führungskräfte, wie oben bereits ausgeführt, auf den Handlungsoptionen beruhen, die durch GRC-Einheiten erarbeitet werden. Eine Übertragung der Verantwortung stellt dies aufgrund des Systems nicht dar, denn zur Verantwortung werden letztlich immer die Oberleitung und die GL gezogen. Die vermehrten angelsächsischen Tendenzen im Schweizer Recht und vor allem im Finanzmarktrecht, die als Ausfluss die vollständige Dokumentation aller Sachverhalte mit sich bringen und Sanktionen bis auf die Stufe der Mitarbeiter zulassen, lassen jedoch darauf schließen, dass neuerdings alle Führungskräfte und Mitarbeiter in der Verantwortung sind und bei künftigen Vorfällen viel genauer erwogen werden kann, welche Person zu welchem Zeitpunkt welche Vorschriften eingehalten oder eben missachtet hat. Diese Schaffung von genauen Evidenzen könnte künftig dazu führen, dass bei großen Vorfällen nicht mehr der VRP oder der CEO seine Funktion verlässt, sondern die tatsächlich handelnden Personen.

\section{These 2: Es zählen nach wie vor die Erträge und die Gewinne - GRC ist nachrangig}

Die Gewinne und die Ertragslage sind für privatwirtschaftliche Unternehmen, wie es die meisten Institute in der Finanzbranche sind, existentiell. Existentiell sind aber auch die Einhaltung neuer Vorschriften und insbesondere der richtige Umgang mit Risiken. Die Risiken im Bankgeschäft haben sich dabei von den eigentlichen Primärrisiken, wie es bspw. die Kreditrisiken sind, zu den Reputations- und operationellen Risiken verschoben. Des Weiteren kommt hinzu, dass die Erträge zur Finanzierung und Implementierung neuer regulatorischen Vorschriften zwingend benötigt werden. Falls diese wegfallen, kann das Institut nicht mehr bestehen. Es kann somit gesagt werden, dass die Verkaufsziele und die GRC-Ziele gleich zu gewichten sind: Ohne Einnahmen aus dem Geschäft kann der GRC-Apparat nicht finanziert werden, und ohne einwandfreie Einhaltung von GRC wird die Banklizenz entzogen.

\section{F. Ausblick}

Welches Ausmaß die rechtlichen und regulatorischen Entwicklungen im Finanzmarkt- und generell im Wirtschaftsrecht noch annehmen, kann zum heutigen Zeitpunkt nicht gesagt werden. Fakt ist, dass eine Internationalisierung des Rechts stattfindet und sich Staaten und Finanzinstitute als Auswuchs der Finanzkrise und der Globalisierung neu an Regeln halten müssen, die nicht demokratisch legitimiert sind. Daraus entsteht als Gegenbewegung nationaler Protektionismus und ein Wettbewerb zwischen den staatlichen Regulierungen.

Neue Dokumentationspflichten und Formalismus führen dazu, dass jeder Schritt nachgewiesen werden kann und somit die Verantwortung der Oberleitungsorgane und der GL wohl nicht mehr in der korrekten Anwendung des Geschäfts und der Prozesse steht, sondern in der korrekten Implementierung aller Vorschriften und Dokumentierungsmaßnahmen anhand IT-unterstützter Prozesse. Die Implementierung und Überwachung der neuen Gesetze, Regeln und Vorschriften kostet die Gesamtwirtschaft sowie die einzelnen Institute heute bereits sehr viel Geld und wird es auch künftig tun. Somit kann erahnt werden, dass sich TBTF-Institute massiv verkleinern und heute bereits kleine Institute ihre Geschäftsfelder stark einschränken werden. Das Stellenprofil der Führungskräfte, aber auch der Frontmitarbeiter in der Finanzbranche wird wohl künftig mehr Know-how in den GRC-Themen fordern, wobei das breite und tiefe Wissen über das komplexe Finanzmarktrecht und dessen innerer und äußerer Zusammenhänge einen kritischen Erfolgsfaktor in den Fähigkeiten darstellen wird. Die Regulierungsdichte und die engmaschige Einführung neuer Regeln werden sich mittelfristig voraussichtlich stabilisieren, so dass der Fokus langfristig wieder auf die einzelnen Kunden und die Ausgestaltung eines rentablen Geschäfts gelegt werden kann. Vorfälle wie bspw. die „Panama-Papers" helfen jedoch nicht, den Kundenfokus wieder zu erhalten, sondern können dazu führen, dass weitere Maßnahmen im Bereich des nationalen und internationalen Steuerrechts angedacht werden.

\section{G. Fazit}

Reputationsverluste, Bankeninsolvenzen, Regulierungsbestrebungen, Staatsüberschuldungen - die Finanzkrise 2007/ 2008 hat nicht abschätzbare Auswirkungen auf die globale (Finanz-)Wirtschaft. Die täglichen Berichterstattungen zeigen, dass das Thema immer noch omnipräsent ist. Im Zusammenhang mit dieser Entwicklung werden die Begriffe Governance, Risk und Compliance immer mehr zum Trend: Jedes Unternehmen und vor allem jedes Finanzinstitut braucht eine gute Corporate Governance, muss seine Risiken im Griff haben und hat „compliant“ zu sein. Doch wie gehen die Führungskräfte mit GRC im sich verändernden regulatorischen Umfeld um? Bilden die GRC-Themen überhaupt einen Bestandteil der Führungsaufgabe? Die Lehrmeinungen sowie die Praktikerstimmen zeigen, dass die Auswirkungen der Finanzkrise auf die Führungstätigkeit markant sind. Die Lehre und die Regulatoren bieten unerschöpfliche Quellen über die Themenbereiche. Vollumfängliche Abhandlungen über die neuen regulatorischen Rahmenbedingungen sind jedoch Mangelware, da sich der globale und nationale Überblick im laufenden Wandel als Herausforderung gestaltet.

Der Blick auf die Führungspraxis zeigt, dass auf Stufe der Oberleitungsorgane sowie der Geschäftsleitung eines Instituts Themen wie Corporate Governance, Wertewandel, Kommunikation, Transparenz, Regulierungen und der sog. „tone from the top" sehr wichtige Aspekte sind. Im Bereich der mittleren sowie der unteren Führungsstufe sind die Themen Risiko, Compliance, Reportings und Kontrollen Bestandteile des Führungsalltags. Allen Stufen gemein ist, dass heute viel mehr Zeit für die Umsetzung regulatorischer Vorschriften, die Kontroll- und Überwachungstätigkeiten und die Entscheidung über das Eingehen von Risiken als für andere Führungsthemen aufgewendet wird. Die Führungsentscheide gehen heute vermehrt in Richtung der bewussten Ablehnung er- 
tragsversprechender Geschäfte. Dabei ist das gezielte Aufzeigen von Handlungsoptionen durch die Risiko- oder Compliance-Einheiten essenziell. Aussagen wie Überregulierungstendenzen, Übernahme des angelsächsischen Sanktionsmechanismus, Dokumentationspflichten, Ermüdung am Thema, Überformalismus, fehlender Pragmatismus, essenzieller Kostenblock für ein Institut und nicht alignierte Regulierungen werden in der Führungspraxis und in den Medien laut und da$\mathrm{zu}$ in neusten Dissertationen verdeutlicht. Die Entwicklung wird wohl dahin gehen, dass mittelfristig im internationalen Wettbewerb die nationalen Regulierungen um ihre kritischen Erfolgsfaktoren buhlen und (hoffentlich) langfristig die Kundenbedürfnisse wieder mehr in den Fokus gestellt werden.
Claudia G. Schoch, Master of Science ZFH in Management \& Law, arbeitet als Associate Director im Bereich Business Risk Management bei der UBS Switzerland $A G$ in Zürich.

RAin Prof. Ursula Sury ist Inhaberin und Geschäftsführerin der Anwaltskanzlei Die Advokatur Sury in Luzern, Professorin und Dozentin an der Hochschule Luzern im Fachbereich Wirtschaft sowie dortige Leiterin des Competence Centers Management \& Law und der Studienrichtung Management \& Law.

RAin Dr. Katharina Hastenrath ist Dozentin für Compliance und Compliance Management an der Züricher Hochschule für Angewandte Wissenschaften. 